\title{
Conhecimento local e pesca de maçunim (Anomalocardia flexuosa) no sistema estuarino-lagunar do Roteiro, Alagoas - Brasil
}

\author{
Letícia Salua Maraschin Mottola ${ }^{1,2 *}$ (D), Gianfrancisco Schork ${ }^{3}$ (D), Jotahi Rodrigues Ferreira Pino ${ }^{2}$ (D), \\ Renato de Mei Romero ${ }^{1}$ (D), Daniel de Magalhães Araujo ${ }^{1,2}$ (iD) \\ ${ }^{1}$ Instituto Federal de Alagoas (IFAL), Campus Marechal Deodoro, Programa de Pós Graduação em Tecnologias \\ Ambientais. Rua Lourival Alfredo, 176 - Poeira, CEP 57160-000, Marechal Deodoro, AL - Brasil. \\ ${ }^{2}$ Instituto Federal de Alagoas (IFAL), Campus Satubal, Rua 17 de Agosto S/N, Zona Rural, CEP: 57120000, Satuba, AL \\ - Brasil. \\ ${ }^{3}$ Universidade Federal do Sul da Bahia (UFSB), Laboratório de Recursos Pesqueiros e Aquicultura (LAPAQ). Rodovia \\ Joel Mares, BR 367, Km 10, s/n, Cx. Postal: 108, CEP: 45.8010-000, Porto Seguro, BA - Brasil. \\ * Autor para correspondência: leticiasalua@gmail.com
}

Recebido em 30 de abril de 2020.

Aceito em 21 de setembro de 2020.

Publicado em 30 de setembro de 2020.

Resumo - O sistema estuarino-lagunar do Roteiro, parte central do litoral alagoano, é um ambiente caracterizado por conflitos socioambientais que conjugam a presença de uma unidade de conservação, a alta demanda turística e a pesca de comunidades tradicionais que procuram no bivalve Anomalocardia flexuosa um importante recurso alimentar e de geração de renda. Assim, dentro desse cenário, objetivou-se caracterizar a atividade extrativista de A. flexuosa na laguna. Para tal, no ano de 2019, aplicaram-se 14 questionários semiestruturados aos marisqueiros. A investigação também foi baseada na observação livre das rotinas de pesca. Os resultados mostraram pontos de convergência entre conhecimentos tradicionais e acadêmicos quanto à influência da competição intraespecífica por espaço e alimento para o recrutamento de novos indivíduos e às quedas de produtividade causadas por altos índices de pluviosidade. A mariscagem é feita de forma rudimentar, com o auxílio de um petrecho denominado ticuca. As capturas ocorrem em áreas com grande variabilidade ambiental, baixa energia de onda e sedimento arenoso. O beneficiamento do bivalve é feito no próprio local de desembarque, em ranchos de pesca construídos de forma precária e em condições sanitárias inadequadas. As conchas são descartadas ao redor dos ranchos - cerca de $27.000 \mathrm{~kg}$ de conchas/mês -, modificando a paisagem local.

Palavras-chave: Bivalves. Conhecimento Tradicional. Comunidades Extrativistas. Mariscagem. Pesca Artesanal.

\section{Local knowledge and fishing of maçunim (Anomalocardia flexuosa) in the estuarine-lagoon system of Roteiro, Alagoas - Brazil}

Abstract - The estuarine-lagoon system of Roteiro, a central part of the Alagoas coast, is an
environment characterized by socio-environmental conflicts that combine the presence of a
conservation unit, the high tourist demand, and the fishing of traditional communities that seek
the bivalve Anomalocardia flexuosa as an important food source and income-generating resource.
Thus, within this scenario, the objective was to characterize the extractive activity of A. flexuosa 
in the lagoon. To reach this purpose, in 2019, 14 semi-structured questionnaires were applied to shellfish gatherers. The investigation was also based on free observation of fishing routines. The results showed points of convergence between traditional and academic knowledge regarding the influence of intraspecific competition for space and food for the recruitment of new individuals and the falls in productivity caused by high rainfall rates. The collection is done in a rudimentary manner, with the aid of a tool called ticuca. Catches occur in areas with great environmental variability, low wave energy, and sandy sediment. The processing of the bivalve is carried out on the landing site, on poorly constructed fishing ranches, and in inadequate sanitary conditions. The shells are discarded around the ranches - about 27,000 kg shells/month -, changing the local landscape.

Key-words: Bivalves. Traditional Knowledge. Extractive Communities. Bivalve Mollusks Gathering. Artisanal Fishery.

\section{Conocimiento local y captura de almeja (Anomalocardia flexuosa) en el sistema de estuarios y lagunas de Roteiro, Alagoas - Brasil}

Resumen - El sistema de estuarios y lagunas de Roteiro, parte central de la costa de Alagoas, es un entorno caracterizado por conflictos socioambientales que combinan la presencia de una unidad de conservación, la alta demanda turística y la pesca de comunidades pesqueras tradicionales que buscan en el bivalvo Anomalocardia flexuosa un importante recurso alimentario y generador de ingresos. Así, dentro de este escenario, el objetivo fue caracterizar la actividad extractiva de A. flexuosa en la laguna. Para ello, en 2019, se aplicaron 14 cuestionarios semiestructurados a marisqueros. La investigación también se basó en la observación libre de las rutinas de pesca. Los resultados mostraron puntos de convergencia entre el conocimiento tradicional y académico sobre la influencia de la competencia intraespecífica por espacio y alimentos para el reclutamiento de nuevos individuos y las caídas en la productividad causadas por los altos niveles de lluvia. La captura de marisco se realiza de forma rudimentaria, con la ayuda de una trampa llamada "ticuca" y ocurren en áreas con gran variabilidad ambiental, baja energía de las olas y sedimento arenoso. El procesamiento del bivalvo se hace en el propio local del desembarque, en chozas de pesca construidas de manera precaria y en condiciones sanitarias inadecuadas. Las conchas son desechadas alrededor de las chozas - cerca de $27.000 \mathrm{~kg}$ de conchas/mes, cambiando el paisaje local.

Palabras clave: Bivalvos. Conocimiento Tradicional. Comunidades Extractivas. Captura de Bivalvos. Pesca Artesanal.

\section{Introdução}

Conhecido popularmente como maçunim, vôngole ou berbigão, Anomalocardia flexuosa (Linnaeus, 1767) é uma espécie de molusco bivalve da família Veneridae com ampla distribuição, ocorrendo desde as Antilhas até o Uruguai (Rios 1994). Presente em toda costa brasileira, é encontrado com maior abundância em zonas entremarés com águas rasas de baixa turbulência e pouco material em suspensão, ficando normalmente enterrado superficialmente em substratos arenosos e arenolodosos (Narchi 1972).

É descrita como uma espécie eurihalina, euritérmica e com resistência a condições prolongadas de anoxia (Leonel et al. 1983; Schaeffer-Novelli 1980; Hiroki 1977; Boehs et al. 2008). Características que, somadas ao seu rápido crescimento, permitem a formação de bancos naturais com biomassas 
significativas em habitats com grande variabilidade ambiental, como enseadas, baías e desembocaduras de estuários (Arruda-Soares et al. 1982; Cantera 1991; Pezzuto e Echternacht 1999).

No Brasil, a relação de A. flexuosa com a pesca está historicamente associada ao extrativismo de bancos naturais. Registros de sambaquis formados predominantemente por suas conchas são associados a áreas ocupadas por sociedades pré-colombianas (Boffi 1979; Scheel-Ybert et al. 2003), mostrando a forte relação desse recurso com o modo de vida das comunidades litorâneas. Porém, apesar da produção ainda ser realizada de forma exclusivamente artesanal e extrativista, sem qualquer tecnologia de cultivo associada, soma cerca de $18 \%$ da produção de moluscos bivalves do país (MPA 2011), fornecendo importante fonte de alimento e de geração de renda para comunidades pesqueiras em toda costa brasileira (Nishida et al. 2004; Dias et al. 2007; Monteles et al. 2009; Silva-Cavalcanti e Costa 2011; Pezzuto e Souza 2015).

Nesse mesmo sentido, no estado de Alagoas, o maçunim aparece como um dos principais moluscos bivalves capturados. Entre as localidades que direcionam suas capturas para a espécie, destacam-se os municípios de Barra de São Miguel e Roteiro, ambos localizados nas margens do sistema estuarino-lagunar do Roteiro, parte central do litoral alagoano. Região esta que é marcada por conflitos socioambientais que conjugam a presença da Reserva Ecológica de Manguezais da Lagoa do Roteiro - RESEC do Roteiro - (Alagoas 1987), a crescente atividade antrópica impulsionada pela vocação turística de seu litoral (Seplag/AL 2018), a vulnerabilidade social de sua população (ADHB 2013) e a presença de comunidades pesqueiras tradicionais.

Assim, dentro desse contexto, o presente estudo teve como objetivo investigar os modos de produção de A. flexuosa no sistema-estuarino lagunar do Roteiro e gerar informações sobre a espécie, considerando o conhecimento local dos pescadores como principal fonte de informação. Destarte, pretende fornecer subsídios que possam auxiliar na construção do ordenamento da pesca na região, e, consequentemente, do uso do espaço e da unidade de conservação ali estabelecida.

\section{Material e métodos}

Os trabalhos de campo foram realizados no sistema estuarino-lagunar do Roteiro, localizado na região central do litoral de Alagoas - coordenadas 09 50' 0" de latitude Sul e 35 57' 30" de longitude Oeste (Figura 1) -, durante o ano de 2019. Primeiramente, quatro visitas exploratórias foram feitas para reconhecimento geral da área, contato inicial com pessoas de referência que atuam na pesca dos bivalves, conhecimento mínimo da atividade extrativista em questão, bem como para identificação do principal ponto de desembarque - local para o qual os esforços da investigação então se direcionaram. 
Figura 1. Localização da área de estudo, sistema estuarino-lagunar do Roteiro, Alagoas, Brasil.

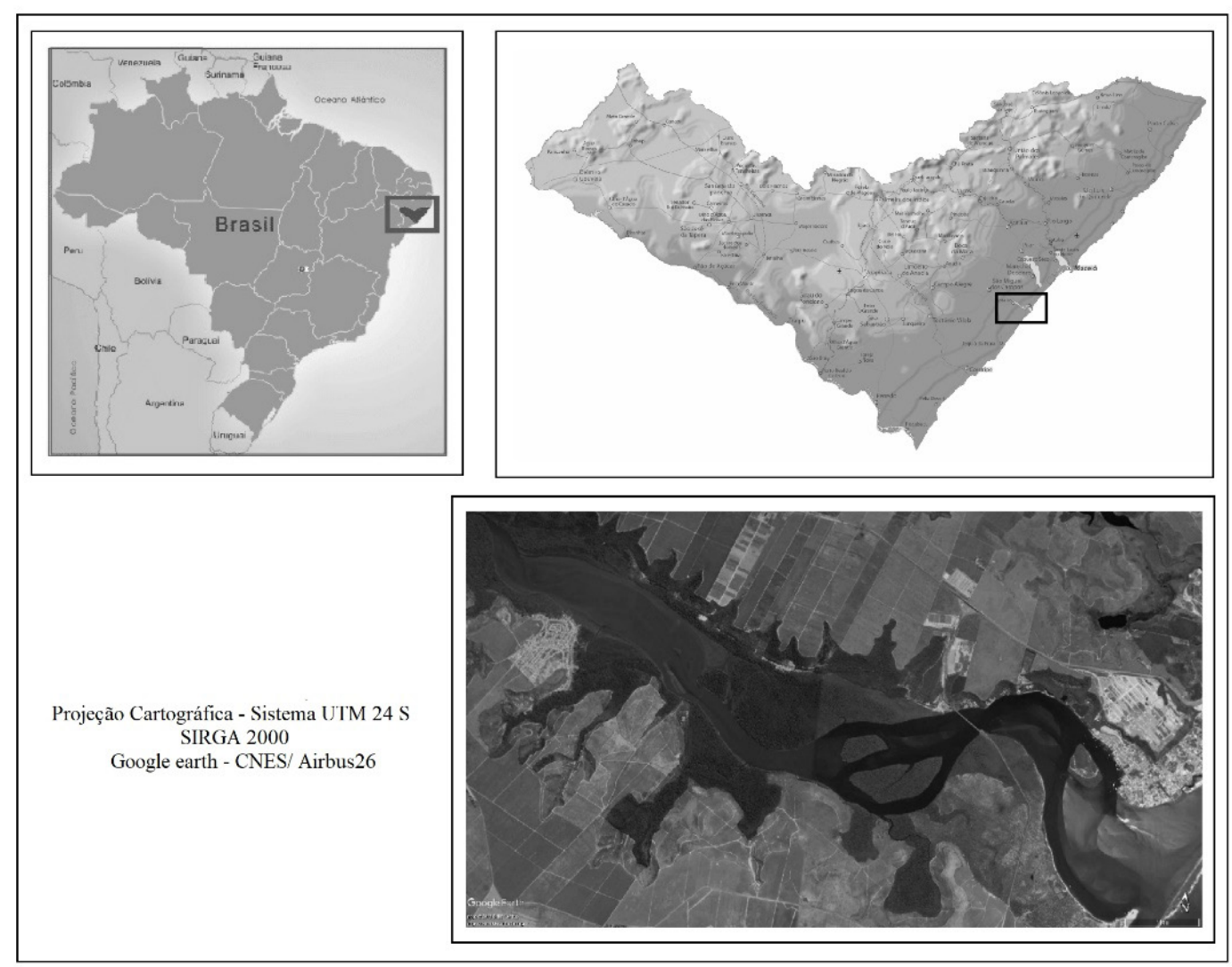

Após esse entendimento primário sobre a mariscagem na região, foi elaborado um questionário semiestruturado, baseado na literatura disponível sobre pesca e com o objetivo de investigar, dentre outras questões: modo de realização das pescarias; esforço médio empregado; forma de beneficiamento, armazenamento, comercialização e descarte de resíduos dos bivalves capturados; e conhecimentos ecológicos locais sobre o recurso em questão tais como fatores que influenciam a produtividade pesqueira.

Antes de cada entrevista, os pesquisadores se identificavam, explicando o intuito da pesquisa e de que forma seriam utilizados os dados. Nesse momento, o Termo de Consentimento Livre Esclarecido (TCLE) era lido e assinado, esclarecendo-se possíveis dúvidas sobre o estudo. Todas as etapas do estudo foram aprovadas pelo Comitê de Ética da Universidade Federal de Alagoas ( ${ }^{\circ}$ 15019819.5.0000.5013).

As primeiras entrevistas foram realizadas com aqueles pescadores identificados nas primeiras incursões ao local eque afirmaram visar o maçunim como principal recurso pesqueiro. Posteriormente, foi solicitado a esses que indicassem seus pares para que também fossem entrevistados e assim sucessivamente, método denominado "bola de neve" (Bailey 1982). Os questionários foram aplicados em dias e horários diversos, de forma a melhor oportunizar o encontro com os pescadores de acordo com suas rotinas de trabalho. Assim, os entrevistados foram abordados principalmente durante o desembarque ou beneficiamento do pescado nos ranchos de pescas, nas proximidades da laguna ou em suas residências. 
Para adequada caracterização do ambiente onde a atividade é exercida, os sítios preferenciais de pesca foram assinalados pelos entrevistados em um mapa da região. Ainda que a atividade seja de pesca artesanal, extrativista, e de base familiar, os pescadores se utilizam de materiais para facilitar a obtenção do molusco, reduzindo o esforço de pesca. Esses petrechos utilizados pelos pescadores foram registrados e descritos, analisados através de observação in loco, com medição do tamanho de malhas entre nós opostos, e registrados através de fotografias.

De forma complementar, a pesquisa também foi baseada na observação livre, por meio do acompanhamento das atividades rotineiras dos pescadores, e nos relatos fornecidos pelos pescadores ao longo da pesquisa, conforme Bruyne et al. (1977). Todas as informações consideradas relevantes foram registradas em áudios gravados pelos pesquisadores ao final dos dias de entrevistas, em um diário de campo e, quando cabível, ilustradas por fotografias.

Todas as estimativas de capturas foram feitas com base nas declarações feitas pelos pescadores. A Captura por Unidade de Esforço (CPUE) ( $\mathrm{kg} \mathrm{hora}^{-1}$ de pesca) foi estimada dividindo-se a produção total diária pelo esforço total, em horas de pesca, conforme a equação: CPUE = QME / TP. Sendo que nesta: $\mathrm{QME}=$ Quantidade $(\mathrm{kg})$ média de parte edível de maçunim oriunda de um dia de pesca; $\mathrm{TP}=$ Tempo (horas) de pesca durante o período de um dia. Já para determinar as capturas mensais em quilogramas (CM), considerou-se o número médio de dias trabalhados por semana, sendo o mês composto por 4,35 semanas. Assim: $\mathrm{CM}=\mathrm{QME} \times \mathrm{n} \mathrm{n}^{\circ}$ dias de pesca por semana $\mathrm{x} 4,35$.

A renda mensal (RM) dos marisqueiros foi calculada a partir da captura mensal (CM) de parte edível $(\mathrm{kg})$ e do preço por quilograma $(\mathrm{R} \$$ ) que os pescadores alegaram obter com a venda do produto em três diferentes situações (valores mínimos, médios e máximos). Dessa forma: $\mathrm{RM}=\mathrm{CM} \times \mathrm{R}$. Para estimar o peso total das capturas (animal inteiro: parte edível mais concha) e o peso das conchas descartadas, utilizou-se o índice de rendimento (IC) de 13,77\% apontado pela literatura (Lavander et al. 2011).

Os aspectos qualitativos da pesquisa foram analisados sob a abordagem emicista/eticista, comparando-se os conhecimentos tradicionais/êmicos com os encontrados na literatura acadêmica (Feleppa 1986).

\section{Resultados}

\section{Reconhecimento geral da área}

As visitas exploratórias permitiram identificar o principal ponto de desembarque das capturas de maçunim feitas na laguna do Roteiro: bairro Zé Nunes, em margem próxima à desembocadura do rio, no município de Barra de São Miguel. Cabe destacar que, além desse, outros dois pontos de desembarque foram identificados: Povoado da Palateia, também no município de Barra de São Miguel, e orla do município de Roteiro. Nestes, porém, o maçunim aparece como recurso complementar, sendo o sururu (Mytella falcata) o principal bivalve capturado.

\section{Atividade extrativista}

Por meio do reconhecimento do local e das informações fornecidas pelos extrativistas, estimouse a presença de 18 pessoas atuando na coleta de A. flexuosa no bairro Zé Nunes. Assim considerado, 
no total, 14 questionários foram aplicados (4 pescadores se negaram em responder), percentual que representa aproximadamente $77,78 \%$ da população estimada.

Segundo as marcações assinaladas pelos entrevistados, a pesca ocorre em uma porção da laguna que se situa no entorno e no interior da RESEC do Roteiro, iniciando em área anterior à abertura do canal e abrangendo um maior número de pontos próximos à desembocadura (Figura 2).

Figura 2. Áreas de pesca utilizadas pelos coletores de A. flexuosa no sistema estuarino-lagunar do Roteiro, AL. Em verde, a área da Reserva Ecológica de Roteiro; em amarelo, as áreas de pesca; em vermelho, o ponto de desembarque. Fonte: Adaptado de Google Earth, 08/10/2019.

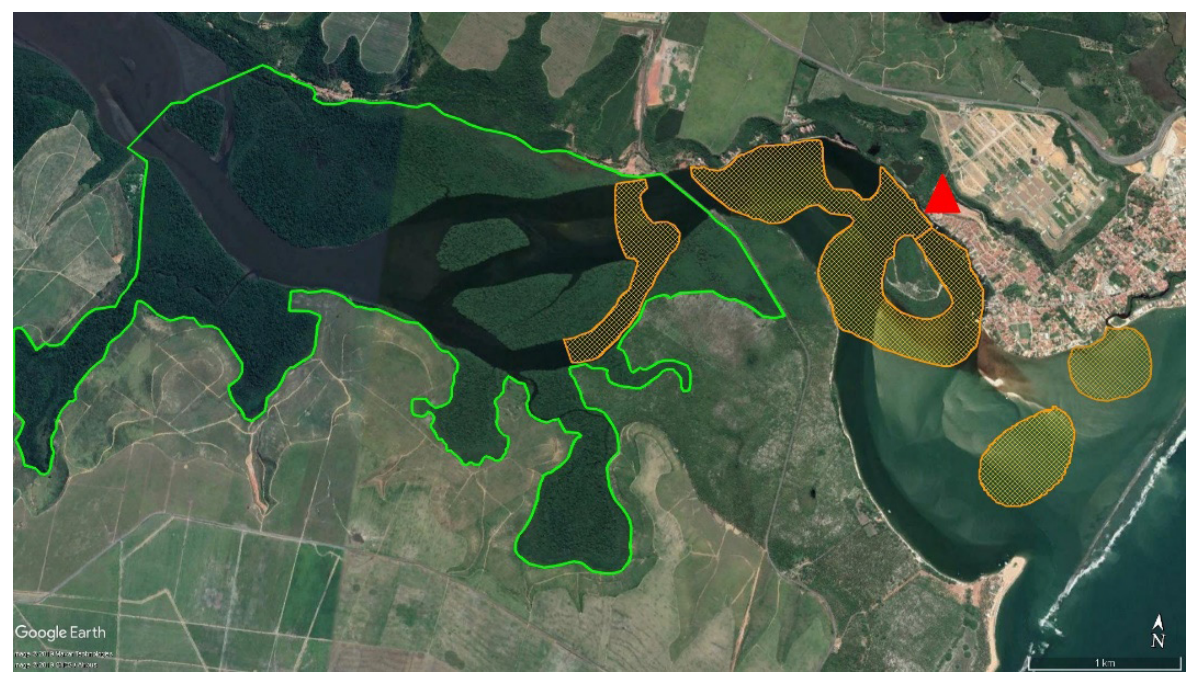

A retirada dos organismos é realizada com o auxílio de um petrecho denominado ticuca (Figura 3) e, apesar dos pescadores utilizarem malhas que variam entre 10 e $20 \mathrm{~mm}$ entre nós opostos, o tamanho mais comumente encontrado em campo foi o de $12 \mathrm{~mm}$. O petrecho é encomendado a um artesão da localidade e custa, aproximadamente, $\mathrm{R} \$ 100,00$. Apenas um entrevistado, uma mulher de idade avançada, disse fazer a retirada dos bivalves diretamente com as mãos, alegando não possuir a força necessária para utilizar o citado artefato.

Figura 3. Petrecho denominado ticuca utilizado para auxiliar a retirada do maçunim do substrato no sistema estuarino-lagunar do Roteiro, AL. Fonte: Schork G, 2019.
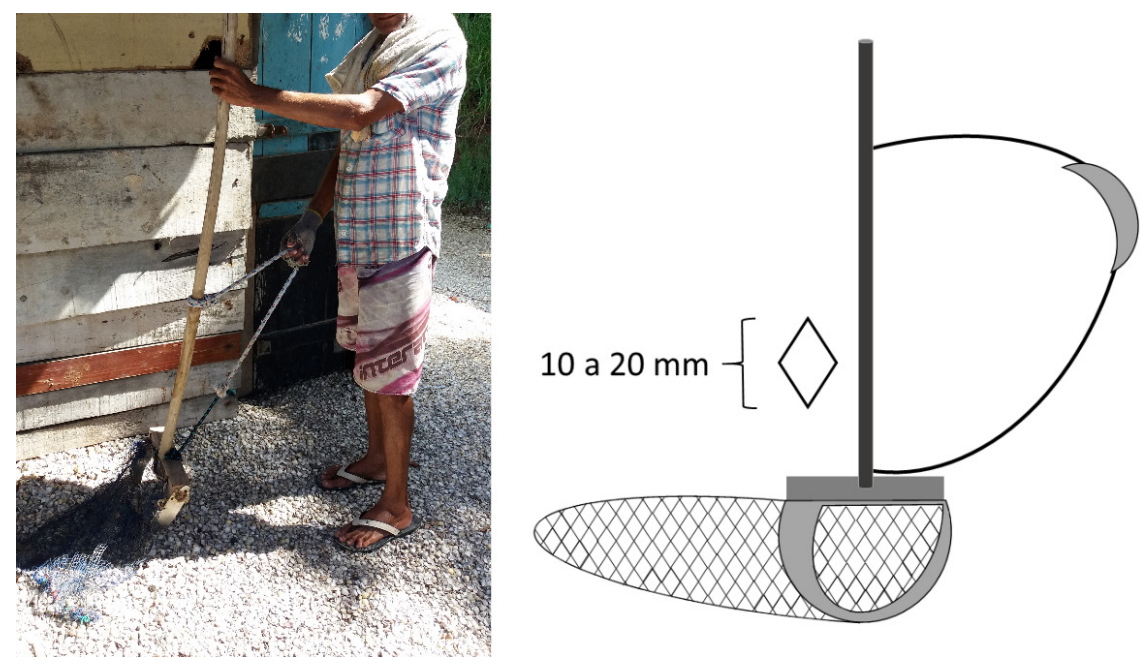
A observação das rotinas de pesca mostrou que a coleta do maçunim é realizada em áreas de pequena profundidade da laguna (até aproximadamente 1,5 metros), preferencialmente em horários de baixa insolação (antes das 10 horas). A escolha do local de coleta é feita de forma diferenciada por cada um dos catadores. Alguns deles declararam possuir pontos fixos de pesca (28,57\%), repetindoos sem qualquer intervalo, enquanto outros trabalham de acordo com a profundidade determinada pela maré $(21,43 \%)$ e/ou procuram pelos locais com maiores quantidades $(21,43 \%)$ (Tabela 1$)$. Todos os pescadores utilizam barcos para acessar o local de pesca, porém, apenas 58,33\% possuem motor (“motor de rabeta"). Na maioria das vezes $(83,33 \%)$, o barco é de propriedade do entrevistado. A maré morta $(50,00 \%)$ e a maré baixa $(42,86 \%)$ foram citadas como condições ambientais benéficas para a prática da atividade.

Tabela 1. Estratégia de escolha do local de pesca citados pelos catadores de maçunim da laguna do Roteiro, AL.

\begin{tabular}{cccc}
\hline Aspectos & Respostas & n & $\%$ \\
\hline & Pontos fixos & 4 & 28,57 \\
& Conforme maré, prefere locais mais rasos & 3 & 21,43 \\
$\begin{array}{c}\text { Estratégia de escolha } \\
\text { do local de pesca }\end{array}$ & Procura por locais com maiores quantidades & 3 & 21,43 \\
& Aleatório & 2 & 14,29 \\
& Procura por locais com maiores organismos & 1 & 7,14 \\
& Faz rodízio & 1 & 7,14 \\
\hline
\end{tabular}

Quanto à sazonalidade, a pesca ocorre em todos os meses do ano, entretanto, segundo os relatos dos pescadores mais experientes, assim considerados aqueles que possuem mais de 10 anos na atividade, o inverno proporciona a captura de organismos maiores, enquanto o verão os melhores preços de venda. Estes também alegaram que consideram a retirada dos organismos adultos como essencial para manter a produtividade do local uma vez que acreditam que o espaço é limitante para o crescimento de novos indivíduos. A partir de seu entendimento, também atribuíram a queda na produtividade do inverno de 2017 aos altos índices de pluviosidade na região.

A coleta é feita, em média, durante 4,08 dias da semana, empregando-se 7,46 horas diárias de esforço (Tabela 2). Já para o beneficiamento dos organismos, a frequência semanal é de 4,32 dias e 7,27 horas diárias. A quantidade de parte edível obtida por dia, segundo os pescadores, varia entre cinco e $40 \mathrm{~kg}$, gerando, em média, 13,54 kg/dia. Assim, a CPUE estimada foi de 1,82 kg hora-1 de pesca, enquanto, excluindo-se os dados do único pescador que coleta o maçunim sem o uso da ticuca, a CPUE foi 1,92 kg hora-1 de pesca. 
Tabela 2. Tempo médio empregado na pesca e beneficiamento do maçunim e quantidade média de parte edível $(\mathrm{kg})$ capturada por dia pelos pescadores da laguna do Roteiro, AL.

\begin{tabular}{cccc}
\hline Aspectos & Mínimo & Máximo & Média \\
\hline No de dias semanais que trabalha na coleta & 7,27 & 7 & 4,08 \\
No de horas diárias que trabalha na coleta & 3 & 12 & 7,46 \\
No de dias semanais que trabalha no beneficiamento & 2 & 7 & 4,32 \\
No de horas diárias que trabalha no beneficiamento & 3 & 12 & 7,27 \\
Quantidade de parte edível $(\mathrm{kg})$ capturada por dia & 5 & 40 & 13,54 \\
\hline
\end{tabular}

Com isso, considerando um total de 18 pescadores, estimou-se que a comunidade estudada retire do local aproximadamente $244 \mathrm{~kg} /$ dia e $4.322 \mathrm{~kg}$ de parte edível/mês. Os mesmos cálculos, considerando o peso total dos organismos com concha, resultaram em $1.770 \mathrm{~kg} / \mathrm{dia}$ e $31.386 \mathrm{~kg} / \mathrm{mês}$. Assim, pode-se inferir que o processamento do molusco por esta comunidade gere aproximadamente $1.526 \mathrm{~kg}$ de conchas/dia e $27.064 \mathrm{~kg}$ de conchas/mês, resíduo descartado indevidamente, como tratado a seguir.

De forma complementar, registra-se a informação de que a presença do peixe-boi (Trichechus manatus manatus) foi citada pelos pescadores como situação comum na laguna, não sendo apontada como prejudicial à pesca. Da mesma forma, não houve relatos de situações de hostilidade com a espécie.

\section{Beneficiamento e comercialização}

O beneficiamento ocorre no próprio local de desembarque, em ranchos de pesca construídos de forma precária (Figura 4A). O maçunim é cozido, normalmente em galões de tinta reutilizados (Figura 4B), e sua concha é retirada com auxílio de uma peneira (Figura 4C). Entre os entrevistados, $42,86 \%$ disseram beneficiar após o retorno da pescaria, ainda no mesmo dia, enquanto os demais realizam o processo no dia seguinte. Em todos os ranchos, o cozimento dos organismos utiliza lenha como combustível uma vez que o preço do gás é considerado economicamente inviável pelos pescadores. Segundo os pescadores, é necessário, em média, uma lata de 18 litros de organismos com concha para se obter um quilo de carne. $\mathrm{O}$ armazenamento do produto é feito em refrigeradores próprios, somente um entrevistado disse utilizar refrigerador de terceiro, instalados em cada rancho de pesca, até a sua venda. 
Figura 4. Ranchos de pesca construídos de forma precária (A), recipientes improvisados nos quais o maçunim é cozido (B), pescador realizando o beneficiamento com auxílio de peneira (C) e descarte de conchas de maçunim em frente aos ranchos de pesca no sistema estuarino-lagunar do Roteiro, AL (D). Fonte: Mottola LSM, 2019.

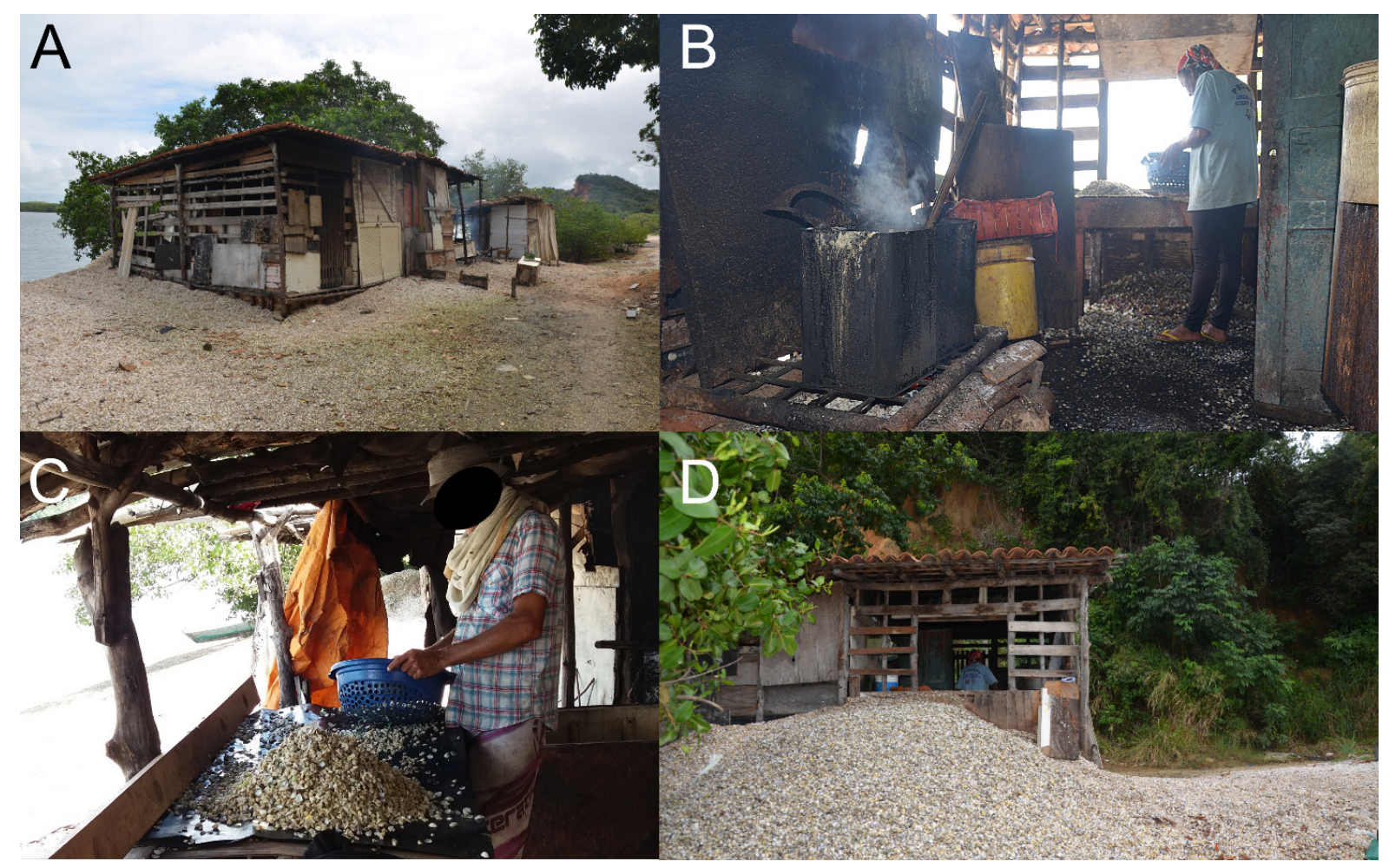

Apenas um entrevistado afirmou vender sua produção somente para o consumidor final, sendo mais comum a venda exclusiva para atravessadores (42,86\%) ou para ambos (50\%). Nesse último caso, ainda que os pescadores aproveitem oportunidades de vendas diretas, a negociação, de forma geral, segue concentrada com o atravessador. Assim, a maior parte da produção é comercializada uma ou duas vezes na semana e direcionada para um único comprador que se desloca até os ranchos de pesca. Segundos os relatos, alguns turistas também aparecem no ponto de desembarque, mas a falta de um local mais receptivo aos compradores dificulta que essas melhores oportunidades de venda se desenvolvam. Combinando essas possibilidades, como local de venda, o ponto de desembarque foi citado por $43,48 \%$ dos entrevistados e a residência do próprio pescador por $47,83 \%$ deles. Feiras e peixarias foram citadas apenas em $8,70 \%$ das entrevistas.

O preço médio declarado do quilo de venda variou entre $\mathrm{R} \$ 7,00$ e $\mathrm{R} \$ 10,00$ no verão e entre $\mathrm{R} \$ 5,00$ e R \$8,00 no inverno - preço imposto pelo atravessador. Algumas pequenas vendas feitas por encomenda e direto para o consumidor final podem atingir valores entre $R \$ 10,00$ e $R \$ 20,00$, mas não somam grande volume. Considerando três diferentes cenários ( $R \$ 5,00 ; R \$ 8,00 ; R \$ 10,00)$, a renda média mensal estimada resultou, respectivamente, em R $\$ 1.200,00, \mathrm{R} \$ 1.921,00$ e R \$2.401,00.

As conchas, assim como os materiais utilizados para o cozimento, são descartadas no próprio local de desembarque, ao redor dos ranchos (Figura 4D). No momento, não existe qualquer aproveitamento para o material. Porém, em anos anteriores, os catadores afirmaram que, esporadicamente, ocorreram vendas das conchas para um comprador de Arapiraca (AL) com a finalidade de uso em ração animal. Algumas vendas para aterros também já foram realizadas, entretanto, sem continuidade. Indagados 
sobre o possível uso das conchas para artesanato, os catadores alegaram que o retorno financeiro é insignificante e, por isso, não dedicam tempo a essa atividade.

\section{Discussão}

Apesar da coleta de A. flexuosa ser prática comum ao longo de toda costa brasileira (SilvaCavalcanti \& Costa 2011), certas peculiaridades marcam a pescaria em cada localidade. Como uma das principais formas de manifestação dessas idiossincrasias, tal como explorado por Nishida et al. (2006), figuram os petrechos de pesca utilizados para a retirada do molusco do sedimento. No caso, o uso da ticuca no estado de Alagoas, nas lagunas Mundaú e Manguaba, já havia sido relatado na década de 1980 no inventário feito por Silva e Barros (1987), o que mostra que as tecnologias artesanais de captura do maçunim utilizadas na região há mais de três décadas atrás ainda hoje são mantidas. De forma paralela, o uso das mãos, ainda que com menor poder de captura, também segue como alternativa para os indivíduos com pouca força física. O uso de barcos de pequeno comprimento, fabricação simples e sem motor exprime a ideia de a pesca de bivalves ser desenvolvida com uso de baixa tecnologia e, consequentemente, pouco investimento financeiro.

Quanto à seletividade do petrecho, o tamanho dos organismos capturados varia de acordo com o tamanho da malha utilizada. Uma vez que é predominante o uso da malha $12 \mathrm{~mm}$, abre-se a discussão sobre o possível direcionamento da pesca para indivíduos ainda jovens. A literatura aponta que as capturas devem ocorrer sobre indivíduos com comprimento acima de $20 \mathrm{~mm}$, tamanho no qual já possuem o grau de desenvolvimento gonadal necessário para sua reprodução (Arruda-Soares et al. 1982). Esse mesmo comprimento é o tamanho mínimo legalmente estabelecido para a captura na Reserva Extrativista Marinha do Pirajubaé, em Santa Catarina, um dos poucos locais no Brasil com ordenamento pesqueiro específico para A. flexuosa (Pezzuto e Souza 2015). Assim, combinar estudos futuros que investiguem a seletividade do petrecho local e a biologia reprodutiva da espécie é um passo que não deve ser ignorado antes da implementação de qualquer medida intervencionista que pretenda limitar o tamanho da malha permitido como uma alternativa para garantir a reprodução do maçunim no local.

As áreas de pesca assinaladas pelos entrevistados confirmaram a descrição de habitat da espécie: zonas com grande variabilidade ambiental e com baixa energia de onda (Narchi 1972, Arruda-Soares et al. 1982; Cantera 1991; Pezzuto e Echternacht 1999). A comparação dessas áreas com estudos geomorfológicos sobre o sistema estuarino-lagunar do Roteiro (Silva 2001) indicou que a presença de A. flexuosa é encontrada em classes texturais predominantemente arenosas.

A metodologia de escolha dos locais e momentos de coleta do molusco evidenciou a posição dos pescadores como sujeitos com comportamento fortemente vinculado às condições do meio ambiente. Distintivamente de algumas pescarias nas quais a modernização da tecnologia de captura permite que o homem se imponha, em maior grau, frente às condições ambientais, aqui, a técnica rudimentar empregada limita o acesso ao recurso-alvo a zonas de pequena profundidade e, por conseguinte, vincula a ação dos pescadores às variações da maré. Os locais escolhidos também têm relação com a acessibilidade - os grupos com menor vigor físico e/ou com barcos movidos a força humana optam por pontos mais próximos e rasos.

A pesca em pontos fixos, denominados como crôas (bancos arenosos expostos na maré baixa), e a ausência da prática do rodízio como forma autogerida de manutenção dos estoques parece indicar 
que, provavelmente, a capacidade de recuperação dos bancos de A. flexuosa ainda é suficiente para repor as taxas de capturas diárias no local. A partir dessa hipótese, na comparação dos conhecimentos êmicos com os acadêmicos, um primeiro ponto de convergência pode ser observado: trata-se do entendimento dos pescadores de que a retirada dos organismos adultos impulsiona o recrutamento da população.

Segundo a literatura, as altas densidades populacionais das classes mais velhas levam a uma forte competição intraespecífica por espaço e alimento, o que, consequentemente, acaba por regular o tamanho das populações (Monti et al. 1991; Pezzuto e Echternacht 1999; Boehs et al. 2008). Como resultado, sua extração libera espaço nos bancos arenosos e permite o estabelecimento de indivíduos jovens. Entretanto, importante observar que, conforme discute Silva-Cavalcanti e Costa (2011), um acréscimo na densidade não pode ser traduzido necessariamente como um aumento da biomassa disponível. Além disso, seguem os autores, a extração excessiva pode levar a uma diminuição do tamanho médio dos organismos coletados e, consequentemente, prejudicar a qualidade da carne peso e palatabilidade. Mais uma vez, para confirmar tais hipóteses, recomenda-se que estudos sobre densidade e biomassa populacional sejam conduzidos na laguna.

Em consonância com o trabalho de Oliveira et al. (2011) no litoral do Pernambuco, que observou maior abundância de indivíduos adultos durante o inverno, outra informação convergente é a relação estabelecida entre a estação do ano e a captura de organismos maiores. Deve-se explicar que, para os pescadores locais, existem apenas duas estações, as quais são definidas de acordo com a abundância de chuvas, sendo o verão seco e o inverno chuvoso. O conhecimento dos pescadores a respeito da variação pluviométrica anual é validado pela classificação oficial de Köppen, que enquadra a região com clima "As", ou seja, tropical, quente e com chuvas de outono e inverno (Barros et al. 2012).

Em relação às observações climáticas sobre o inverno de 2017, a alta pluviosidade na região é confirmada pelos dados oficiais de meteorologia (SEMARH 2017). No mesmo sentido do entendimento dos pescadores, a redução nas densidades populacionais de A. flexuosa após períodos com chuvas intensas é uma associação que também pode ser encontrada em estudos feitos a partir de monitoramentos científicos (Monti et al. 1991; Boehs et al. 2008; Maia et al. 2018). Conforme discutem, além da redução brusca da salinidade poder causar choques osmóticos, a ressuspensão de material do fundo causada pelas chuvas também é um fator determinante para regular o sucesso do estabelecimento da espécie. De acordo com Narchi (1972), o bivalve apresenta sifões curtos e fundidos, com tentáculos simples, que não barram de forma eficiente a entrada de sedimentos para o seu interior e, por isso, em ambientes com elevada carga de material em suspensão é comum a asfixia e morte de organismos.

A média de frequência semanal e horas diárias despendidas nas atividades de pesca e póspesca mostra que a maioria dos pescadores do local dedica seus dias de forma integral à atividade. Logicamente, o tempo de esforço empregado em cada etapa tem relação com as divisões de tarefas estabelecidas para o beneficiamento do produto. Parte dos catadores trabalha sozinho e, por isso, precisa dividir o tempo entre as atividades de pesca e beneficiamento. Outros catadores trabalham em regime de parceria, geralmente com familiares, o que permite que aumentem o esforço na retirada dos organismos. Além da clara relação entre a quantidade capturada por dia e o tempo empregado na pescaria, também se deve considerar o vigor físico do indivíduo para explicar o largo intervalo declarado para as capturas diárias. A comum presença de pescadores acima de 50 anos de idade, 
do sexo feminino, com limitações de saúde e, inclusive, portadores de deficiências (registro de um indivíduo cego) é característica notável da comunidade estudada.

Quanto às capturas, os valores de CPUE encontrados se assemelham aos valores encontrados por Silva-Cavalcanti \& Costa (2009) analisando conjuntamente diferentes petrechos de coleta do maçunim (1,2 a 2,2 kg carne/h), indicando que a ticuca tem um poder de captura equiparável a outras metodologias de pesca da espécie.

Notadamente, as atividades pós-pesca constituem um ponto chave para a qualidade e valor do produto. A precariedade dos ranchos de pesca e das condições de beneficiamento colocam em risco a qualidade higiênico-sanitária da carne oferecida e afastam o contato com o consumidor final, que prefere comprar o produto sob a aparente segurança que oferecem os demais elos da cadeia produtiva de pescados (peixarias, restaurantes, etc.). A falta de associativismo também obstaculiza determinadas iniciativas, principalmente quanto ao comércio do produto, que seriam mais facilmente alcançáveis quando geridas em conjunto. Nesse cenário, a figura do atravessador ainda permanece como um gargalo para o escoamento da produção local, situação que é reiteradamente relatada na pesca de pequena escala como uma das causas das baixas margens de lucro obtidas pela venda do pescado (Seixas et al. 2011; Partelow et al. 2018).

Melhorias na infraestrutura hoje instalada, com o intuito de aproximar o consumidor final, não parecem uma alternativa que possa ser viabilizada pelos próprios pescadores, considerandose a margem de lucro advinda da pesca, fato que coloca o poder público na figura de protagonista como agente transformador dessa realidade. Também nesse sentido, a oferta de oficinas que visem o aprimoramento técnico dos pescadores - a exemplo de áreas como melhorias nas práticas de beneficiamento do pescado, gestão financeira e associativismo - poderiam ser alternativas para elevar o lucro atual sem demandar aumento do esforço pesqueiro. Estratégias de construção de capital humano e social que já são bem discutidas no contexto da pesca artesanal (Castello et al. 2009; Gutiérrez, et al. 2011; Cole et al., 2018; Frawley, et al. 2019).

Ainda que a comunidade estudada seja aparentemente de pequeno porte, a estimativa de peso total de conchas descartadas mensalmente demonstra a dimensão do resíduo gerado. Uma possibilidade é incentivar a ressignificação das conchas como um produto de valor econômico e auxiliar a abertura de canais de comunicação que facilitem o contato da comunidade pesqueira com empresários de áreas com potencial uso do material (Chierighini et al. 2011; Souza et al. 2014; Silva et al. 2019).

\section{Conclusões}

A mariscagem no local ainda hoje é feita de forma artesanal, com uso de petrechos rudimentares, indicando que as tecnologias de produção utilizadas pelas comunidades pesqueiras que vivem da coleta de A. flexuosa pouco se alteraram ao longo do tempo na costa alagoana. Resultado que destaca a continuidade da transmissão do conhecimento ao longo de diferentes gerações, comportamento característico de comunidades reconhecidas como tradicionais.

A convergência encontrada entre os conhecimentos acadêmicos e êmicos reitera a importância de se considerar o conhecimento tradicional como fonte de informação valiosa na formação de bancos de dados sobre a espécie e na construção de ordenamentos da atividade pesqueira e da região. 
Apesar da comunidade estudada ser aparentemente pequena, figura como protagonista no uso do espaço e dos recursos da laguna. Além de seus ranchos de pesca ocuparem área integrada com a zona urbana e em contato com outras atividades econômicas, principalmente a turística, o acúmulo de conchas oriundas da atividade extrativista é um elemento visivelmente importante na transformação da paisagem local. Assim, a pesca e os pescadores de A. flexuosa devem ser elementos a serem sempre considerados em futuras ações interventivas na laguna.

\section{Agradecimentos}

Agradecemos à Pró-Reitoria de Pesquisa, Pós-Graduação e Inovação (PRPPI) do Instituto Federal de Alagoas (IFAL) pela bolsa de Iniciação Científica (EDITAL nº 04 PRPI/IFAL de 10 de maio de 2018) concedida ao terceiro autor. Também agradecemos aos pescadores e pescadoras da laguna do Roteiro pela receptividade e participação.

Participação dos autores: LSMM - Participou de todas as fases do projeto, autora da dissertação de mestrado que gerou os dados do manuscrito; GS - elaboração do projeto, contato com as comunidades; pesquisas de campo e revisão final do artigo; JRFP - Participou ativamente nas pesquisas de campo; RMR - coorientador da dissertação de mestrado. Participou da elaboração do projeto e da revisão final do artigo; DMA - orientador da dissertação de mestrado, participou de todas as fases do projeto.

Aprovaçãoética elicenças: Aprovado pelo Comitê de Ética da Universidade Federal de Alagoas (nº 15019819.5.0000.5013).

Disponibilidade dos dados: Dados não estarão disponíveis em base ou repositores.

Fomento: Uma bolsa de Iniciação Científica (EDITAL no 04 PRPI/IFAL de 10 de maio de 2018) foi concedida ao terceiro autor.

Conflito de Interesses: Não há qualquer conflito de interesse.

\section{Referências}

Alagoas. 1987. Decreto $n^{\mathbf{0}}$ 32.355, de 03 de junho de 1987. Cria a Reserva Ecológica de Manguezais da Lagoa do Roteiro e dá outras providências. Diário Oficial do Estado de Alagoas. https://www.ima.al.gov.br/wp-content/ uploads/2015/03/Decreto-nb0-32.355_87.pdf

Arruda-Soares H, Schaeffer-Novelli Y, Mandelli-Jr J. 1982. “Berbigão” Anomalocardia brasiliana (Gmelin, 1791), bivalve comestível da região da Ilha do Cardoso, Estado de São Paulo, Brasil: aspectos biológicos de interesse para a pesca comercial. Boletim do Instituto de Pesca, 9(único):21-38. https://www.pesca.sp.gov.br/sumario_9_21-38.pdf

Atlas do Desenvolvimento Humano do Brasil (ADHB). 2013. Barra de São Miguel e Roteiro, Al. http://www.atlasbrasil. org.br. Acesso em: 15 mai. 2018.

Bailey KD. 1982. Methods of Social Research. The Free Press, New York. 553 p.

Barros AHC, Araujo-Filho JC, Silva AB, Santiago GACF. 2012. Climatologia do estado de Alagoas. Embrapa SolosBoletim de Pesquisa e Desenvolvimento. INFOTECA-E Embrapa, Recife. 32 p.

Boehs G, Absher TM, Cruz-Kaled AC. 2008. Ecologia populacional de Anomalocardia brasiliana (Gmelin, 1791) (Bivalvia, Veneridae) na Baía de Paranaguá, Paraná, Brasil. Boletim do Instituto de Pesca, 34(2):259-270. https://www. pesca.sp.gov.br/34_2_259-270.pdf

Boffi AV. 1979. Moluscos brasileiros de interesse médico e econômico. Pimenta de Mello, Rio de Janeiro. 376 p. 
Bruyne P, Herman J, Schoutheete M. 1977. Dinâmica da pesquisa em ciências sociais: os polos da prática metodológica. Francisco Alves, Rio de Janeiro. 252 p.

Cantera JR. 1991. Shallow-water venerid clams (Bivalvia: Veneridae) from the pacific coast of Colombia. Veliger, 34(1):78-84.

Castello L, Viana JP, Watkins G, Pinedo-Vasquez M, Luzadis VA. 2009. Lessons from integrating fishers of arapaima in small-scale fisheries management at the Mamirauá Reserve, Amazon. Environmental management, 43(2), 197-209. DOI: $10.1007 /$ s00267-008-9220-5

Chierighini D, Bridi R, Rocha AD, Lapa KR. 2011. Possibilidades do uso das conchas de moluscos. In: 3rd International Workshop Advances in Cleaner Production. São Paulo, p.1-5.

Cole SM, McDougall C, Kaminski AM, Kefi, AS, Chilala, A, Chisule G. 2018. Postharvest fish losses and unequal gender relations: drivers of the social-ecological trap in the Barotse Floodplain fishery, Zambia. Ecology and Society, $23(2): 18$. DOI: 10.5751/ES-09950-230218

Dias TLP, Rosa RS, Damasceno LCP. 2007. Aspectos socioeconômicos, percepção ambiental e perspectivas das mulheres marisqueiras da Reserva de Desenvolvimento Sustentável Ponta do Tubarão (Rio Grande do Norte, Brasil). Gaia Scientia, 1(1): 25-35. https://periodicos.ufpb.br/index.php/gaia/article/view/2225/1953

Feleppa R. 1986. Emics, etics, and social objectivity. Current Anthropology, 27(3):243-254. https://www.jstor.org/ stable/2742879

Frawley TH, Finkbeiner EM, Crowder, LB. 2019. Environmental and institutional degradation in the globalized economy. Ecology and Society, 24(1):7. DOI: 10.5751/ES-10693-240107

Gutiérrez, N. L., R. Hilborn, e O. Defeo. 2011. Leadership, social capital and incentives promote successful fisheries. Nature 470:386-389. DOI: 10.1038/nature09689

Hiroki K. 1977. On the resistance of isolated bivalve gill pieces to oxygen deficiency and hydrogen sulphide. Boletim de Fisiologia Animal, 1:9-20.

Lavander HD, Júnior LDOC, Oliveira RL, Silva-Neto SR, Galvez AO, Peixoto SR. 2011. Biologia reprodutiva da Anomalocardia brasiliana (Gmelin, 1791) no litoral norte de Pernambuco, Brasil. Revista Brasileira de Ciências Agrárias, 6(2):344-350. DOI: 10.5039/agraria.v6i2a1139

Leonel RMV, Magalhães ARM, Lunetta JE. 1983. Sobrevivência de Anomalocardia brasiliana (Gmelin, 1791) (Mollusca:Bivalvia), em diferentes salinidades. Boletim de Fisiologia Animal, 7:63-71.

Maia AML, Medeiros E, Henry-Silva G.G. 2018. Distribution and density of the bivalve Anomalocardia brasiliana in the estuarine region of Northeastern Brazil. Brazilian Journal of Biology, 78(1): 32-40. DOI: 10.1590/1519-6984.02316

Ministério da Pesca e Aquicultura (MPA). 2011. Boletim Estatístico da Pesca e Aquicultura 2011. Brasília: MPA. 60 p. https://www.icmbio.gov.br/cepsul/images/stories/biblioteca/download/estatistica/est_2011_bol_bra.pdf

Monteles JS, Castro TCS, Viana DCP, Conceição FS, França VL, Funo ICSA. 2009. Percepção sócio-ambiental das marisqueiras no município de Raposa - MA. Revista Brasileira de Engenharia de Pesca, 4(2):34-35. DOI: 10.18817/ repesca.v4i2.141

Monti D, Frenkiel L, Moueza M. 1991. Demography and growth of Anomalocardia brasiliana (Gmelin) (Bivalvia: Veneridae) in a mangrove, in Guadeloupe (French West Indies). Journal of Molluscan Studies, 57(2):249-257. DOI: 10.1093/mollus/57.2.249

Narchi W. 1972. Comparative study of the functional morphology of Anomalocardia brasiliana (Gmelin, 1791) and Tivela mactroides (Born, 1778) (Bivalvia, Veneridae). Bulletin of Marine Science, 22(3):643-670. https://www.ingentaconnect. com/contentone/umrsmas/bullmar/1972/00000022/00000003/art00006\# 
Nishida AK, Nordi N, Alves RRN. 2004. Abordagem etnoecológica da coleta de molusco no litoral paraibano. Tropical Oceanography, 32(1):53-68. DOI: 10.5914/tropocean.v32i1.5034

Nishida AK, Nordi N, Alves RRN. 2006. Mollusc gathering in Northeast Brazil: An ethnoecological approach. Human Ecology, 34(1):133-145. DOI: 10.1007/s10745-005-9005-x

Oliveira IB, Amorim A, Lavander H, Peixoto S, Gálvez AO. 2011. Spatial and temporal distribution of the shellfish Anomalocardia brasiliana (Gmelin, 1791) on Mangue Seco beach, Pernambuco, Brazil. International Journal of Aquatic Science, 2:68-79. http://www.journal-aquaticscience.com/article_73583_c187f4f4ca93892b46725f7d423a9763.pdf

Partelow S, Glaser M, Arce SS, Barboza RSL, Schlüter A. 2018. Mangroves, fishers, and the struggle for adaptive comanagement: applying the social-ecological systems framework to a marine extractive reserve (RESEX) in Brazil. Ecology and Society 23(3):19. DOI: 10.5751/ES-10269-230319

Pezzuto PR, Echternacht AM. 1999. Avaliação de impactos da construção da Via Expressa SC-Sul sobre o berbigão Anomalocardia brasiliana (Gmelin, 1791) (Mollusca: Pelecypoda) na Reserva Extrativista Marinha do Pirajubaé (Florianópolis, Brasil). Atlântica, 21:105-119.

Pezzuto PR, Souza DS. 2015. A pesca e o manejo do berbigão (Anomalocardia brasiliana) (Bivalvia: Veneridae) na Reserva Extrativista Marinha do Pirajubaé, SC, Brasil. Desenvolv. Meio Ambient. 34: 169-189. DOI: 10.5380/dma.v34i0.39758

Rios, E. 1994. Seashells of Brazil. Rio Grande: FURG. 492 p.

Schaeffer-Novelli, Y. 1980. Análise populacional de Anomalocardia brasiliana (Gmelin, 1791), na Praia do Saco da Ribeira, Ubatuba, Estado de São Paulo. Boletim do Instituto Oceanográfico, 29(2):351-355. DOI: 10.1590/S037355241980000200070

Scheel-Ybert R, Eggers S, Wesolowski V, Petronilho CC, Boyadjian CH, Deblasis PAD, Gaspar MD. 2003. Novas perspectivas na reconstituição do modo de vida dos sambaquieiros: uma abordagem multidisciplinar. Revista Arqueologia, 16:109-137.

Secretaria de Estado do Planejamento (SEPLAG/AL). 2018. Perfil Municipal de Barra de São Miguel. Ano 4, no 4 (2013). Secretaria de Estado do Planejamento, Gestão e Patrimônio, Maceió.

Secretaria do Meio Ambiente e dos Recursos Hídricos (SEMARH). 2017. Precipitação mensal de 2017. Maceió/AL. http://www.semarh.al.gov.br/tempo-e-clima/analises-tecnicas/precipitacao-mensal

Seixas CS, Kalikoski DC, Almudi T, Batista VS, Costa AL, Diogo HL, Ferreira BP, Futemma CRT, Moura RL, Ruffino ML, Salles RD, Thé. APG 2011. Gestão compartilhada do uso de recursos pesqueiros no Brasil: elementos para um programa nacional. Ambiente \& Sociedade 14(1):23-44. DOI: 10.1590/S1414-753X2011000100003

Silva APL. 2001. Estudos Geomorfológico e sedimentológico do sistema estuarino-lagunar do Roteiro-Alagoas. Dissertação de mestrado, Universidade Federal de Pernambuco, Recife.

Silva CS, Barros JBP. 1987. Inventário da malacofauna do complexo lagunar Mundaú/Manguaba, Alagoas. Boletim de Estudos das Ciências do Mar, 6:65-74.

Silva-Cavalcanti JS, Costa MF. 2009. Fisheries in protected and non-protected areas: is it different? The case of Anomalocardia brasiliana at tropical estuaries of Northeast Brazil. Journal of Coastal Research, Special Issue 56(2): 1454-1458. https://www.jstor.org/stable/25738030

Silva-Cavalcanti JS, Costa MF. 2011. Fisheries of Anomalocardia brasiliana in tropical estuaries. Pan-American Journal of Aquatic Sciences, 6(2):86-99. https://panamjas.org/pdf_artigos/PANAMJAS_6(2)_86-99.pdf

Silva HT, Mesquita-Guimarães J, Henriques B, Silva F, Fredel M. 2019. The potential use of oyster shell waste in new value-added by-product. Resources, 8(1):1-15. DOI: 10.3390/resources 8010013 
Souza RG, Sant’anna FSP, Fredel MC, Alarcon OE. 2014. Emprego das conchas residuais da maricultura na fabricação de revestimento cerâmico autoclavado. Cerâmica Industrial, 19(4):27-30. https://ceramicaindustrial.org.br/ article/587657577f8c9d6e028b4808/pdf/ci-19-4-587657577f8c9d6e028b4808.pdf 